Publ. RIMS Kyoto Univ.

Vol. 6 (1970/71), 515-543

\title{
On Besov Spaces of Functions Defined in General Regions*
}

By

\author{
Tosinobu Muramatu
}

\section{$\S 1 . \quad$ Introduction}

In this paper we discuss certain spaces of distributions on an open set $\Omega$ in the $n$-space $\mathbb{F}^{n}$ : Besov spaces. These spaces are first introduced and investigated by $O$. V. Besov [1] for $\Omega=\mathbb{R}^{n}$ (see also [15]), for a general region they are studied by V. P. Il'in [6]. We here investigate in particular their relation to the theory of mean interpolation spaces due to Lions-Peetre [10]. M. H. Taibleson [17] discussed also for the case $\Omega=\mathbb{R}^{n}$ in a different point of view. For the case $\Omega=\mathbb{R}^{n}$ an operator theoretical approach is available, [5], [7], [10], [13], [19].

To describe our results we need some notations. For a measure space $(M, \mu)$ by $L^{p}(M ; X)$ we denote the space of all strongly measurable functions $u$ with value in a Banach space $X$ such that

$$
\|u\|_{L^{p(M: X)}}= \begin{cases}\left\{\int_{M}\|u(\xi)\|_{X}^{p} d \mu(\xi)\right\}^{1 / p} & (1 \leqq p<\infty), \\ \text { ess. } \sup \|u(\xi)\|_{X} & (p=+\infty),\end{cases}
$$

is finite, where $\|f\|_{X}$ means the norm of $f$ in $X$. In particular, $L^{p}(M)$ $=L^{p}(M ; \mathbb{C})$. By $L_{*}^{p}\left(\mathbb{x}^{\mathrm{p}}+; X\right)$ and $L_{*}^{p}\left(\mathbb{R}^{n} ; X\right)$ we denote the $L^{p}$-space for $\left(\mathbb{R}^{+}, t^{-1} d t\right)$ and $\left(\mathbb{R}^{n},|x|^{-n} a^{\prime} x\right)$, respectively; while for $\Omega \subset \mathbb{R}^{n}$ by $L^{p}(\Omega)$ we denote the $L^{p}$-space with respect to the usual Lebesgue measure $d x=d x_{1} \cdots d x_{n}$. Let $\alpha=\left(\alpha_{1}^{\prime}, \cdots, \alpha_{n}\right)$ be a multi-index of nonnegative integers. Then we write

$$
D^{\alpha}=D_{1}^{\alpha_{1} \ldots} D_{n}^{\alpha_{n}}, \quad D_{j}=\partial / \partial x_{j},
$$

Received November 2. 1970.

*Thesis presented to the University of Tokyo. 


$$
\begin{aligned}
& x^{\alpha}=x_{1}^{\alpha_{1}} \cdots x_{n}^{\alpha_{n}}, \\
& |\alpha|=\alpha_{1}+\cdots+\alpha_{n}, \quad \alpha !=\alpha_{1} ! \cdots \alpha_{n} !, \\
& \left(\begin{array}{l}
\alpha \\
\beta
\end{array}\right)=\frac{\alpha !}{\beta !(\alpha-\beta) !} \quad \text { if } \quad \alpha \geqq \beta \geqq 0 \\
& =0 \quad \text { otherwise, }
\end{aligned}
$$

here $\alpha \geqq \beta$ means $\alpha_{j} \geqq \beta_{j}$ for $j=1,2, \cdots, n$. For $\Omega \subset \mathbb{R}^{n} C^{m}(\Omega)$ means the space of $m$-times continuously differentiable functions on $\Omega ; \mathscr{B}^{m-}(\Omega)$ the space of functions $\in C^{m-1}(\Omega)$ whose all derivatives of order up to $(m-1)$ are bounded and whose all $(m-1)$-th derivatives are uniformly Lipschitz continuous. $W_{p}^{m}(\Omega)$, called the Sobolev space, is the space of distributions $f$ on $\Omega$ whose (distributional) derivatives of order up to $m$ belong to $L^{p}(\Omega)$, with norm

$$
\|f\|_{W_{p}^{m}(\Omega)}=\sum_{|\alpha| \leqq m}\left\|D^{\alpha} f\right\|_{L^{p}(\Omega)}
$$

$B_{p, q}^{m+\tau, j}(\Omega)$, where $j$ is a positive integer, $0<\tau<j$, called the Besov space, is the space of $f \in W_{p}^{m}(\Omega)$ for which the norm

$$
\|f\|_{B_{p, q}^{m+\tau, j}(\Omega)}=\|f\|_{W_{p}^{m}(\Omega)}+|f|_{B_{p, q}^{m+\tau, j}(\Omega)}
$$

is finite, where

$$
\begin{aligned}
& |f|_{B_{p, q}^{m+\tau, j}(\Omega)} \\
& =\sum_{|\alpha|=m}\left\|\left\{\left\|\sum_{i=0}^{j}\left(\begin{array}{l}
j \\
i
\end{array}\right)(-1)^{i} f(x+i y)\right\|_{L^{p}\left(\Omega_{j, y}\right)}|y|^{-\tau}\right\}\right\|_{L_{*}^{q}\left(\boldsymbol{R}^{n}\right)}, \\
& \Omega_{j, y}=\bigcap_{i=0}^{j}(\Omega-i y) .
\end{aligned}
$$

We denote also

$$
|f|_{W_{p}^{m}(\Omega)}=\sum_{|\alpha|=m}\left\|D^{\alpha} f\right\|_{L^{p}(\Omega)}
$$

For convenience we shall make use of the following terminology:

Definition. An open set $\Omega \supset \mathbb{R}^{n}$ satisfy the condition $C\left(T_{0}, m\right)$ if there is a vector-valued function $\Psi(x) \in \mathscr{B}^{m-}\left(\mathbb{R}^{n}\right)$ and a positive number $T_{0}$, including $T_{0}=+\infty$, such that

$$
x+t z+t \Psi(x) \in \Omega
$$


for any $x \in \Omega,|z| \leqq 1$ and $0<t<T_{0}$ (cone condition).

It will be seen below that the Besov space $B_{p, q}^{m+\tau, j}(\Omega)$ is dependent on the sum $\lambda=m+\tau, p$ and $q$, and not dependent on the choice of $j$, $m$ and $\tau$, if $\Omega$ satisfies the cone condition. Hence ve define as follows: When $\lambda$ is a positive fractional number

$$
B_{p, q}^{\lambda}(\Omega)=B_{p, q}^{m+\tau, 1}(\Omega),
$$

where $\lambda=m+\tau, 0<\tau<1, m$ is an integer. When $\lambda$ is a positive integer,

$$
B_{p, q}^{\lambda}(\Omega)=B_{p, q}^{(\lambda-1)+1,2}(\Omega) .
$$

Then morm \|\|$_{B_{p, q}^{\lambda}}$ and the semi-norm ||$_{B_{p, q}^{\lambda}}$ are defined analogously.

Now, we state our main results.

Theorem 1.1. (Interpolation inequalities) Let $\Omega$ be an open sets satisfying the condition $C\left(T_{0}, i\right)$. Then the following inequalities hold for $0<T<T_{0}$ with constant $C$ independent of $T$ and $f:$ (i) if $f \in W_{p}^{m}(\Omega)$,

$$
\begin{gathered}
T^{k}|f|_{W_{p}^{k}(\Omega)} \leqq C\left\{T^{m}|f|_{W_{p}^{m}(\Omega)}+\|f\|_{L^{p}(\Omega)}, \quad(0<k<m)\right. \\
T^{k+\sigma}|f|_{B_{p, q}^{k+\sigma, i}(\Omega)} \leqq C\left(T^{\sigma}+1\right)\left\{T^{m}|f|_{W_{p}^{m}(\Omega)}+\|f\|_{L^{p}(\Omega)}\right\}, \\
(0<k+\sigma<m ; 0<\sigma<i) \\
T^{m}|f|_{B_{p, \infty}^{(m-k)+k, i}(\Omega)} \leqq C\left(T^{k}+1\right)\left\{T^{m}|f|_{W_{p}^{m}(\Omega)}+\|f\|_{L^{p}(\Omega)}\right\}, \\
(0<k<i ; k \leqq m)
\end{gathered}
$$

(ii) if $f \in B_{p, q}^{m+\tau, j}(\Omega), 0<\tau<j, \quad 0<(2 j-1) \quad T<T_{0}$,

$$
\begin{aligned}
& T^{k}|f|_{W_{p}^{k}(\Omega) \leqq C} \leqq\left\{T^{m+\tau}|f|_{B_{p, q}^{m+\tau, j}(\Omega)}+\|f\|_{L^{p}(\Omega)}\right\}, \\
&(0<k<m+\tau) \\
& T^{k+\sigma}|f|_{B_{p, q}^{k+\sigma, i}(\Omega)} \leqq C\left(T^{\sigma}+1\right)\left\{T^{m+\tau}|f|_{B_{p, q}^{m+\tau, j}(\Omega)}+\|f\|_{L^{p}(\Omega)}\right\} ; \\
&(0<k+\sigma \leqq m+\tau ; 0<\sigma<i)
\end{aligned}
$$

(iii) if $f \in B_{p, 1}^{(m-k)+k, j}(\Omega), \quad 0<k<j, \quad k \leqq m, \quad 0<(2 j-1) T<T_{0}$,

(1. 10) $\quad T^{m}|f|_{W_{p}^{m}(\Omega)} \leqq C\left\{T^{m}|f|_{B_{p, 1}^{(m-k)+k, j_{(\Omega)}}}+\|f\|_{L^{p}(\Omega)}\right\}$.

Consequently, if $0<\tau<i$ and $\lambda=m+\tau$,

$$
B_{p, q}^{\lambda}(\Omega)=B_{p, q}^{m+\tau, i}(\Omega)
$$


with equivalent norms, and for $\lambda^{\prime}>m>\lambda^{\prime \prime}>0$

$$
\begin{aligned}
& B_{p, q}^{\lambda \prime}(\Omega) \subset W_{p}^{m}(\Omega) \subset B_{p, q}^{\lambda \prime \prime}(\Omega), \\
& B_{p, 1}^{m}(\Omega) \subset W_{p}^{m}(\Omega) \subset B_{p, \infty}^{m}(\Omega),
\end{aligned}
$$

with continuous injections.

Furthermore, the norms (1.1) and (1.2) are equivalent to the norms

$$
|f|_{W_{p}^{m}(\Omega)}+\|f\|_{L^{p}(\Omega)}
$$

and

$$
|f|_{B_{p, q}^{m+\tau, j}(\Omega)}+\|f\|_{L^{b}(\Omega)}
$$

respectively.

The factors $\left(T^{\sigma}+1\right),\left(T^{k}+1\right)$ in $(1.6) \sim(1.9)$ are removable if we may take $\Psi(x)$ to be a constant vector.

By means of another method Il'in proved analogous inequalities, but his condition on $\Omega$, though very close to that of us, is somewhat complicated, in the author's opinion.

Theorem 1.2. (Approximation theorem) For any open set $\Omega$, $C^{\infty}(\Omega) \cap W_{p}^{m}(\Omega)$ and $C^{\infty}(\Omega) \cap B_{p, q}^{m+\tau, j}(\Omega)$ are dense in $W_{p}^{m}(\Omega)$ and $B_{p, q}^{m+\tau, j}(\Omega)$, respectively.

Combining two theorems, we have

Corollary. If $\Omega$ satisfies the condition $C\left(T_{0}, j\right)$, then $W_{p}^{m}(\Omega)$ and $B_{p, q}^{m+\tau, j}(\Omega), 0<\tau<j$, are identical with the completions of the sets of $C^{\infty}(\Omega)$-functions $f$ whose norm given by $\left(1.1^{\prime}\right)$ and $\left(1.2^{\prime}\right)$ is finite with respect to those norms, respectively.

Our proof for this theorem is the same as that given by MeyersSerrin [11] for Sobolev spaces, however for Resov spaces we need some preparations.

Next, we shall explain mean interpolation spaces. Let $X, Y$ be Banach space contained in a Hausdorff linear topological space $\mathscr{X}$. By $W(p, \xi, X ; q, \eta, Y)$ we denote the space $L_{*}^{p, \xi}\left(\mathbb{R}^{+} ; X\right) \cap L_{*}^{q, \eta}\left(\mathbb{R}^{+} ; Y\right)$ with norm $\|u\|_{W}=\max \left\{\|u\|_{L_{*}^{p, \xi}\left(\boldsymbol{R}^{+} ; X\right)},\|u\|_{L_{*}^{q, \eta^{\prime}}\left(\boldsymbol{R}^{+} ; Y\right)}\right\}$, where $L_{*}^{p, \xi}\left(\mathbb{R}^{+} ; X\right)$ 
$=\left\{u(t) ; t^{\xi} u(t) \in L_{*}^{p}\left(\boldsymbol{R}^{+} ; X\right)\right.$ with norm $\left\|t^{\xi} u(t)\right\|_{L_{*}^{p}\left(\boldsymbol{R}^{+} ; X\right)}$. The mean interpolation space $S(p, \xi, X ; q, \eta, Y)$ is the space of elements $f$ in $X+Y$ such that

$$
f=\int_{0}^{\infty} u(t) \frac{d t}{t}, \quad u \in W(p, \xi, X ; q, \eta, Y)
$$

with norm

$$
\|f\|_{s}=\inf \left\{\|u\|_{W(p, \xi, X: q, \eta, Y)} ; f=\int \frac{u(t)}{t} d t\right\} .
$$

Here we assume that $\xi \cdot \eta<0$, and $X+Y$ is the space $\{f+g ; f \in X$, $g \in Y\}$ with norm $\|h\|_{X+Y}=\inf \left\{\|f\|_{X}+\|g\|_{Y} ; f \in X, g \in Y, f+g=h\right\}$. It is easily seen that (cf. [10])

$$
\begin{aligned}
& S(p, \lambda \theta, X ; q, \lambda(\theta-1), Y)=S(p, \theta, X ; q, \theta-1, Y) \\
&(0<\theta<1, \lambda \neq 0)
\end{aligned}
$$

with equivalent norms.

Let $\mathscr{H}(X, Y)$ be the space of functions $u(\zeta)$ of the complex variable, holomorphic in the strip $0<\operatorname{Re}<1$ with values in $X+Y$, continuous and bounded in the closed strip $0 \leqq R e \zeta \leqq 1$ with values in $X+Y$, such that $u(i t), t \in \mathbb{R}$, is continuous and bounded with values in $X$, and $u(1+i t), t \in \boldsymbol{R}$, is continuous and bounded with values in $Y$. Under the norm

$$
\|u\|_{\mathscr{H}(X, Y)}=\max \left\{\sup _{t}\|u(i t)\|_{X}, \sup _{t}\|u(1+i t)\|_{Y}\right\}
$$

$\mathcal{H}(X, Y)$ becomes a Banach space. The space $[X, Y]_{\theta}, 0<\theta<1$, is the range of the mapping $u \rightarrow u(\theta), u \in \mathscr{H}(X, Y)$, with the norm

$$
\|f\|_{[X, Y]_{\theta}}=\inf \left\{\|u\|_{\mathcal{H}(X, Y)} ; f=u(\theta)\right\},
$$

(c. f. [4], [8]). With these notations we state

Theorem 1.3. (Interpolation theorem) Let $0<\theta<1$ Then $B_{p, q}^{m \theta}(\Omega)=S\left(q, \theta, L^{p}(\Omega) ; q, \theta-1, W_{p}^{m}(\Omega)\right)$ with equivalent norms. Here we assume that $\Omega$ satisfies the condition $C\left(T_{o}, m\right)$, and that $m$ is a positive integer.

Lions-Peetre [10] proved this theorem for the case $\Omega=\boldsymbol{R}^{n}$, and 
Lions-Magenes [9] proved the case where $\Omega$ is bounded and has a sufficiently smooth boundary and $p=q$ (Sobolev spaces of fractional order) with the aid of the result of Uspenskii [18]; while we shall prove this theorem without any smoothness assumption on the boundary of $\Omega$.

Theorem 1.4. Let $\mu_{1}$ and $\mu_{2}$ be positive numbers and let $0<\theta<1$. Set $\lambda=(1-\theta) \mu_{1}+\theta \mu_{2}$,

$$
\frac{1}{p}=\frac{1-\theta}{p_{1}}+\frac{\theta}{p_{2}}, \quad \frac{1}{q}=\frac{1-\theta}{q_{1}}+\frac{\theta}{q_{2}} .
$$

Then, (i) if $p_{1} \geqq q_{1}$ and $p_{2} \geqq q_{2}$,

$$
B_{p, q}^{\lambda}(\Omega) \supset S\left(q, \theta, B_{p_{1}, q_{1}}^{\mu_{1}}(\Omega) ; q, \theta-1, B_{p_{2}, q_{2}}^{\mu_{2}}(\Omega)\right)
$$

with continuous injection. (ii) If $p_{1} \leqq q_{1}$ and $p_{2} \leqq q_{2}$,

$$
B_{p, q}^{\lambda}(\Omega) \subset S\left(q, \theta, B_{p_{1}, q_{1}}^{\mu_{1}}(\Omega) ; q, \theta-1, B_{p_{2}, q_{2}}^{\mu_{2}}(\Omega)\right)
$$

with continuous injection. (iii)

$$
B_{p, q}^{\lambda}(\Omega)=\left[B_{p_{1}, q_{1}}^{\mu_{1}}(\Omega), \quad B_{p_{2}, q_{2}}^{\mu_{2}}(\Omega)\right]_{\theta}
$$

with equivalent norms. Here we assume that $\Omega$ satisfies the condition $C\left(T_{o}, m\right)$ where $m$ is a positive integer with $m>\mu_{1}, \mu_{2}$.

Grisvard [4] proved this theorem for the case $\Omega=$ IR $^{n}$ by means of an operator theoretical method. An important consequence of the theorem is the following

Corollary. Under the assumption of Theorem 1.4,

$$
B_{p_{1}, q_{1}}^{\mu_{1}}(\Omega) \cap B_{p_{2}, q_{2}}^{\mu_{2}}(\Omega) \subset B_{p, q}^{\lambda}(\Omega),
$$

and

$$
\|f\|_{B_{p, q}^{\lambda}(\Omega)} \leqq C\|f\|_{B_{p_{1}, q_{1}}^{\mu_{1}(\Omega)}}^{1-\theta}\|f\|_{B_{p_{2}, q_{2}}^{\mu_{2}(\Omega)}}^{\theta}
$$

(a generalization of the Gagliardo-Nirenberg inequality), where $C$ is a constant independent of $f$.

The last assertion follows from Theorem 1.4 and a theorem on complex interpolation spaces (cf. [4], [8]).

Our main tool is the integral representation of the form 


$$
\int_{0}^{T} t^{l-1} d t \int K(x, z) f(x+t z+\Psi(x)) d z
$$

which was employed by the author in his study of the imbedding theorem for Sobolev spaces, [12], instead of that of a potential type. The integral (1.16) is very similar to usual potential one, in fact, if $\Psi(x)=0$ it can be transformed into potential type one, however any potential type integral can not be transformed into the form (1.16), therefore our representation is more precise than that of the potential type. Moreover integrals of this form are considerably convenient to deal with, as seen later on. One of the reason is that they can be divided into two steps, the integration with respect to $z$ and that with respect to $t$. The singularity occurs only in the later step.

We end this section by illustrating some examples of open sets satisfying the cone condition.

Example 1. $\boldsymbol{R}^{n}$ satisfies the condition $C(+\infty,+\infty)$ with $\Psi(x)=0$.

Example 2. Let $\Omega$ be a star-shaped bounded region with respect to a ball of radius $r_{0}$ with center at $a$. Then $\Omega$ satisfies the condition $C\left(r_{0},+\infty\right)$ with $\Psi(x)=r_{0}^{-1}(a-x)$.

Example 3. A cone $\Omega=\left\{x ;|x|^{-1} x \in A\right\}$, where $A$ is an open set in the unit spher $S=\{x ;|x|=1\}$ such that for any $\alpha, \beta>0$ and any $x_{1}, x_{2}$ in $A \alpha x_{1}+\beta x_{2} /\left|\alpha x_{1}+\beta x_{2}\right| \in A$, satisfies the condition $C(\infty, \infty)$ with $\Psi(x)=$ constant vector.

\section{\$2. Inequalities for Some Integral Transformations}

We state first the integral representation formula which is proved in $[12]$.

Lemma 2.1. Let $\Omega$ be an open set in $\boldsymbol{R}^{n}$ satisfying the condition $C\left(T_{0}, 1\right)$. Let $\omega(x)$ be a $C^{\infty}$-function such that $\int \omega(x) d x=1$, and its support is contained in the unit ball $B$. Then for any positive integer $m$, any multi-index $\beta$ with $|\beta|<m, 0<T<T_{0}$ and any function $f \in C^{m}(\Omega)$, 
(2.1)

$$
\begin{aligned}
& f^{(\beta)}(x) \\
& =\sum_{|\alpha|=m-|\beta|} \int_{0}^{T} t^{m-|\beta|-1} d t \int \omega_{\alpha}(x, z) f^{(\alpha+\beta)}(x+t z+t \Psi(x)) d z \\
& \quad+T^{-|\beta|} \int \omega_{m, \beta}(x, z) f(x+T z+T \Psi(x)) d z
\end{aligned}
$$

where $f^{(\alpha)}=D^{\alpha} f$

$$
\omega_{\alpha}(x, z)=\frac{(-1)^{|\alpha|}|\alpha|}{\alpha !}(z+\Psi(x))^{\alpha} \omega(z)
$$

and

$$
\omega_{m, \beta}(x, z)=(-1)^{|\beta|} \sum_{|\alpha|<m-|\beta|} \frac{1}{\alpha !} D_{z}^{\alpha+\beta}\left\{(z+\Psi(x))^{\alpha} \omega(z)\right\} .
$$

Corollary. Let $\Omega$ be as above. Then for $|\beta|<m+j, 0<T<T_{0}$ and for any function $f \in C^{m+j}(\Omega)$,

$$
\begin{aligned}
& f^{(\beta)}(x) \\
& =\sum_{|\alpha|=m} \int_{0}^{T} t^{m-|\beta|-1} d t \int K_{j, \alpha, \beta}(x, z) f^{(\alpha)}(x+t z+t \Psi(x)) d z \\
& \quad+T^{-|\beta|} \int \omega_{m+j, \beta}(x, z) f(x+T z+T \Psi(x)) d z,
\end{aligned}
$$

where

$$
K_{j, \alpha, \beta}(x, z)=(-1)^{j} \sum_{\substack{|\gamma|=j \\ \alpha+\gamma=\beta}} C_{\gamma, \alpha, \beta} D_{z}^{\gamma} \omega_{\alpha+\gamma-\beta}(x, z),
$$

and $\left\{C_{\gamma, \alpha, \beta}\right\},|\gamma|=j,|\alpha|=m$, are constants such that

$$
\sum_{\alpha+\gamma=\alpha} C_{\gamma, \alpha, \beta}=1 \quad \text { for a fixed } \tilde{\alpha} \geqq \beta \text {. }
$$

Proof. By (2.1), where $m$ is replaced by $m+j$, we have (2.2) with the aid of the integration by parts, since

$$
\begin{aligned}
& t^{|\gamma|} \int \omega_{\alpha+\gamma-\beta}(x, z) f^{(\alpha+\gamma)}(x+t z+t \Psi(x)) d z \\
& \quad=(-1)^{|\gamma|} \int D_{z}^{\gamma} \omega_{\alpha+\gamma}(x, z) f^{(\alpha)}(x+t z+t \Psi(x)) d z .
\end{aligned}
$$

Remark 2.1. The integrals contained in the right hand side of (2. $\left.1^{\prime}\right)$ are not improper even if $|\beta| \geqq m$ in spite of their appearance.

To prove the boundedness of integral transformations we frequently make use of the following well known fact: 
Lemma 2.2. Let $\left(M_{1}, \mu_{1}\right)$ and $\left(M_{2}, \mu_{2}\right)$ be $\sigma$-finite measure spaces, and let $K(x, y)$ be a measurable function such that

$$
\begin{array}{ll}
\int_{M_{1}}|K(x, y)| d \mu_{1}(x) \leqq C & \text { for a. e. } y \text { in } M_{2} \\
\int_{M_{2}}|K(x, y)| d \mu_{2}(y) \leqq C & \text { for a. e. } x \text { in } M_{1} .
\end{array}
$$

Then the integral operator with kernel $K(x, y)$ is a bounded operator from $L^{p}\left(M_{2}\right)$ into $L^{p}\left(M_{1}\right)$ whose operator norm is not greater than $C$.

We start by stating and proving some basic inequalities concerning integrals similar to that employed in the formula (2.1).

Lemma 2.3. Let $\Omega$ be as in Lemma 2.1.

(i) If we define

$$
U_{0}(t, x)=\int_{B}|f(x+t z+t \Psi(x))| d z
$$

for $f \in L^{p}(\Omega), 0<t<T_{0}$, then

$$
\left\|U_{0}(t, x)\right\|_{L^{p}\left(S_{2}\right)} \leqq a b^{n}\|f\|_{L^{p}\left(\xi_{C}\right)},
$$

where $b=\sup |\Psi(x)|+1$, and $a$ is the volume of the unit ball $B$.

(ii) If we define

$$
\begin{aligned}
U_{j}(t, x)= & \int_{B} d z \int_{(2 j-1) B} \mid \sum_{\nu=0}^{j}(-1)^{\nu}\left(\begin{array}{l}
j \\
\nu
\end{array}\right) \\
& \times j\left(x+t \frac{(j-\nu) z+\nu w}{j}+t \Psi(x)\right) \mid d w
\end{aligned}
$$

for $f \in B_{p, q}^{\tau, j}(\Omega), 0<\tau<j, 0<(2 j-1) t<T_{0}$, then

$$
\left\|U_{j}(t, x)\right\|_{L^{p}(\Omega)} \leqq a b^{n} j^{n} \int_{2 B} F_{j}(t z) d z
$$

where $F_{j}(y)=\left\|\sum_{\nu=0}^{j}\left(\begin{array}{l}j \\ \nu\end{array}\right)(-1)^{\nu} f(x+\nu y)\right\|_{L^{p}\left(\Omega_{j}, y\right)}$,

$$
\left\|U_{j}(t, x)\right\|_{L^{p}(\Omega)} \leqq C t^{\tau}|f|_{B_{p, q}^{\tau, j}(\Omega)}
$$

and 


$$
\begin{aligned}
&\left\{\int_{0}^{T}\left\|U_{j}(t, x) t^{l}\right\|_{L^{p}(\Omega)}^{q} \frac{d t}{t}\right\}^{1 / q} \leqq C T^{l+\tau}|f|_{B_{p, q}^{\tau, j}(\Omega)} \\
& \text { for } 0<(2 j-1) T<T_{0}, l+\tau \geqq 0,
\end{aligned}
$$

where $C$ is a constant dependent only on $n, q, \tau$ and $b$.

Proof. (i) From Jessen's inequality ${ }^{1)}$ it follows that

$$
\begin{aligned}
\left\|U_{0}(t, x)\right\|_{L^{p}(\Omega)} & \leqq\left\|\int_{b B}|f(x+t z)| e(x+t z) d z\right\|_{L^{p}(\Omega)}, \\
& \leqq \int b B\|f(x+t z) e(x+t z)\|_{L^{p}(\Omega)} d z, \\
& \leqq a b^{n}\|f\|_{L^{p}(\Omega)},
\end{aligned}
$$

where $e(x)$ is the characteristic function of $\Omega$.

(ii) Using Jessen's inequality and replacing $w$ by $z+j w$ as variables of integration, we see that

$$
\begin{aligned}
& \left\|U_{j}(t, x)\right\|_{L^{p}(\Omega)} \\
& \quad \leqq\left\|\int_{b B} d z \int_{2 B} j^{n} \prod_{\nu=0}^{j} e(x+t z+t \nu w)\left\{\sum_{\nu=0}^{j}\left(\begin{array}{l}
j \\
\nu
\end{array}\right)(-1)^{\nu} f\left(x+t z+t_{\nu} w\right)\right\} d w\right\|_{L^{p}(\Omega)}, \\
& \quad \leqq \int_{b B} d z \int_{2 B} j^{n}\left\|\sum_{\nu=0}^{j}(-1)^{\nu}\left(\begin{array}{l}
j \\
\nu
\end{array}\right) f(x+t z+t \nu w)\right\|_{L^{p}\left(\Omega_{j,}, t w-t z\right)} d w, \\
& \quad \leqq a b^{n} \int_{2 B} j^{n} F_{j}(t z) d z .
\end{aligned}
$$

By Hölder's inequality we obtain that the right-hand side is not greater than

$$
C\left\{\int_{2 B} F_{j}(t z)^{q}|z|^{-n^{-\tau q}} d z\right\}^{1 / q} \leqq C t^{\top}|f|_{B_{p, q}^{\tau, j}(\Omega)}
$$

Finally, from (2.5) it follows that

$$
\begin{aligned}
\left\|U_{j}(t, x) t^{l}\right\|_{L^{p}(\Omega)} & \leqq C_{1} T^{l+\tau} \int_{2 B} F_{j}(t z) t^{-\tau} d z \\
& =C_{1} T^{l+\tau} \int_{2 t B}(|y| / t)^{n+\tau}\left\{|y|^{-\tau} F_{j}(y)\right\}|y|^{-n} d y,
\end{aligned}
$$

which gives (2.7) with the help of Lemma 2.2, since

$$
\int_{2 t B}\left(\frac{|y|}{t}\right)^{n+\tau} \frac{d y}{|y|^{n}}=\frac{2^{n+\tau} a n}{n+\tau}, \quad \int_{|y| / 2}^{\infty}\left(\frac{|y|}{t}\right)^{n+\tau} \frac{d t}{t}=\frac{2^{n+\tau}}{n+\tau} .
$$

1) Generalized Minkowski inequality. 
Corollary. Under the assumptions of Lemma 2.3 the following inequalities hold for $f \in C^{j}(\Omega) \cap L^{p}(\Omega)$.

(i) If $0<\sigma<k$, then

$$
\begin{array}{r}
\left\|\left\{\left\|U_{j}(t, x)\right\|_{L^{p}(\Omega)} h\left(\frac{|y|}{t}\right)^{k}|y|^{-\sigma}\right\}\right\|_{L_{*}^{q}\left(\boldsymbol{R}^{n}\right)} \\
\leqq\left\{\begin{array}{lll}
C t^{-\sigma}\|f\|_{L^{p}(\Omega)} & \text { for } & j=0, \\
C t^{\tau-\sigma}|f|_{B_{p, q}^{\tau, j}(\Omega)} & \text { for } & j \geqq 1,
\end{array}\right.
\end{array}
$$

where $h(s)=\min (1, s)$.

(ii) If $l+\tau-\sigma \geqq 0,0<(2 j-1) T<T_{0}$ and $0<\sigma<k$, then

$$
\begin{gathered}
\left\|\int_{0}^{T}\right\| U_{j}(t, x)\left\|_{L^{p}(\Omega)} h\left(\frac{|y|}{t}\right)^{k}|y|^{-\sigma} t^{l-1} d t\right\|_{L_{*}^{q}\left(R^{n}\right)} \\
\leqq C T^{l+\tau-\sigma}|f|_{B_{, q}^{\tau}, \dot{j}(\Omega)} \quad \text { for } \quad j \geqq 1 .
\end{gathered}
$$

Proof. (i) A simple calculation shows that

$$
\left\|h\left(\frac{|y|}{t}\right)^{k}|y|^{-\sigma}\right\|_{L_{*}^{q}\left(\boldsymbol{R}^{n}\right)}=\left\{\frac{n a k}{(k-\sigma) \sigma q}\right\}^{1 / q} t^{-\sigma},
$$

which gives the desired inequality (2.8).

(ii) The left-hand side of (2.9) is equal to

$$
\left\|\int_{0}^{T} K(y, t) \varphi(t) t^{-1} d t\right\|_{L_{*}^{q}\left(R^{n}\right)^{\prime}},
$$

where $K(y, t)=(t /|y|)^{\sigma} h(|y| / t)^{k}, \varphi(t)=\left\|U_{j}(t, x) t^{l-\sigma}\right\|_{L^{p}(\Omega)}$. Therefore (2.9) follows from (2.7) and Lemma 2.2.

Lemma 2.4. Let $\Omega$ be an open set satisfying the condition $C\left(T_{0}, 1\right)$. Assume that

$$
K(x, z)=\sum_{|\alpha|=j-1} D_{z}^{\alpha} H_{\alpha}(x, z),
$$

where $D_{z}^{\beta} H_{\alpha}(x, z) \in C\left(\boldsymbol{R}^{n} \times \mathbb{R}^{n}\right)$ for any $\alpha$ with $|\alpha|=j-1$ and for any $\beta$ with $|\beta| \leqq j-1$, and $\operatorname{supp} H_{\alpha} \subset \boldsymbol{R}^{n} \times B, \int H_{\alpha}(x, z) d z=0$ for any $\alpha$ with $|\alpha|=j-1$. Set

$$
V(t, x)=\int K(x, z) f(x+t z+t \Psi(x)) d z .
$$


Then, choosing an appropriate function $\widetilde{K}(x, z, w) \in C\left(\mathbb{R}^{n} \times \mathbb{R}^{n} \times \mathbb{R}^{n}\right)$ with support contained in $\mathbb{R}^{n} \times B \times(2 j-1) B$,

$$
\begin{aligned}
& V(t, x)=\iint \widetilde{K}(x, z, w)\left\{\sum_{\nu=0}^{j}(-1)^{\nu}\left(\begin{array}{l}
j \\
\nu
\end{array}\right)\right. \\
& \left.\quad \times f\left(x+t \frac{(j-\nu) z+\nu w}{j}+t \Psi(x)\right)\right\} d z d w
\end{aligned}
$$

holds for all $f \in C^{1}(\Omega)$.

Proof. It is sufficient to prove the case where $K=D_{z}^{\alpha} H$. Case $j=1$. It is clear from the assumptions that

$$
\begin{aligned}
V(t, x)=\iint K(x, z) \omega(w) & \{f(x+t z+t \Psi(x)) \\
& -f(x+t w+t \Psi(x))\} d z d w
\end{aligned}
$$

where $\omega$ is a $C^{\infty}$ function such that $\int \omega(x) d x=1$ and supp $\omega \subset B$.

Let $K=D_{z}^{\alpha} H=\frac{\partial}{\partial z_{k}} D_{z}^{\beta} H$, and assume that the formula is valid for $K_{1}=D_{z}^{\beta} H$. Then, integrating by parts, we have

$$
\begin{aligned}
V(t, & x)=-t \int K_{1}(x, z) f_{k}(x+t z+t \Psi(x)) d z \quad\left(f_{k}=D_{k} f\right), \\
= & -t \iint \widetilde{K}_{1}(x, z, w) \\
& \times\left\{\sum_{\nu=0}^{j-1}(-1)^{\nu}\left(\begin{array}{c}
j-1 \\
\nu
\end{array}\right) f_{k}\left(x+t \frac{(j-1-\nu) z+\nu w}{j-1}+t \Psi(x)\right)\right\} d z d w, \\
= & -\frac{(j-1)^{n} t}{j^{n}} \iint \widetilde{K}_{1}\left(x, z, \frac{z+(j-1) w}{j}\right) \\
\quad & \times\left\{\sum_{\nu=0}^{j-1}(-1)^{\nu}\left(\begin{array}{c}
j-1 \\
\nu
\end{array}\right) f_{k}\left(x+t \frac{(j-\nu) z+\nu w}{j}+t \Psi(x)\right)\right\} d z d w,
\end{aligned}
$$

(interchange the variables of integration $\left.w \rightarrow \frac{z+(j-1) w}{j}\right)$, $=\iint \widetilde{K}(x, z, w)\left\{\sum_{\nu=0}^{j-1}(-1)^{\nu}\left(\begin{array}{l}j \\ \nu\end{array}\right) f\left(x+t \frac{(j-\nu) z+\nu w}{j}+t \Psi(x)\right)\right\} d z d w$

(note that $\frac{\partial}{\partial z_{k}}\left\{f\left(x+t \frac{(j-\nu) z+\nu w}{j}\right)\right\}=t \frac{j-\nu}{j} f_{k}\left(x+t \frac{(j-\nu) z+\nu w}{j}\right)$ and integrate by part with respect to $\left.z_{k}\right)$, where $\widetilde{K}_{1}(x, z, w)$ is the function whose existence is assured by the inductive assumption, and $\widetilde{K}(x, z, w)$ is given by 


$$
\widetilde{K}(x, z, w)=\frac{(j-1)^{n}}{j^{n}} \frac{\partial}{\partial z_{k}} \widetilde{K}_{1}\left(x, z, \frac{z+(j-1) w}{j}\right) .
$$

Since $\int \widetilde{K}(x, z, w) d z=0$, from the above identity we obtain (2.11).

Lemma 2.5. Let $K(x, z), \widetilde{K}(x, z, w), \Psi(x)$ belong to $\mathscr{B}^{j-}\left(\mathbb{R}^{n} \times \mathbb{R}^{n}\right)$, $\mathscr{B}^{j-}\left(\mathbb{R}^{n} \times \mathbb{R}^{n} \times R^{n}\right), \mathscr{B}^{j-}\left(\mathbb{R}^{n}\right)$, respectively. Then there is a constant $C$ independent of $t, w, x, y, z$ such that

$$
\begin{gathered}
\left|\sum_{\nu=0}^{j}(-1)^{\nu}\left(\begin{array}{l}
j \\
\nu
\end{array}\right) K\left(x+\nu y, z-\frac{\nu y}{t}+\Psi(x)-\Psi(x+\nu y)\right)\right| \\
\leqq C\left\{h\left(\frac{|y|}{t}\right)^{j}+h(|y|)^{j}\right\}, \\
\mid \sum_{\nu=0}^{j}(-1)^{\nu}\left(\begin{array}{l}
j \\
\nu
\end{array}\right) \widetilde{K}\left(x+\nu y, z-\frac{\nu y}{t}+\Psi(x)-\Psi(x+\nu y),\right. \\
\left.w-\frac{\nu y}{t}+\Psi(x)-\Psi(x+\nu y)\right) \mid \\
\leqq C\left\{h\left(\frac{|y|}{t}\right)^{j}+h(|y|)^{j}\right\},
\end{gathered}
$$

where $h(t)=\min (t, 1)$.

Proof. It is not difficult to see that these inequalities follow from the inequality

$$
\begin{aligned}
& \left|\sum_{\nu=0}^{j}(-1)^{\nu}\left(\begin{array}{l}
j \\
\nu
\end{array}\right) \varphi(\nu)\right| \\
& \leqq \int_{0}^{1} \cdots \int_{0}^{1}\left|\varphi^{(j-1)}\left(s_{1}+\cdots+s_{j-1}\right)-\varphi^{(j-1)}\left(s_{1}+\cdots+s_{j-1}+1\right)\right| d s_{1} \cdots d s_{j-1}
\end{aligned}
$$

where $\varphi^{(j-1)}=\frac{d^{j-1} \varphi}{d s^{j-1}}, \varphi \in \mathscr{B}^{j-}(\boldsymbol{R})$.

Combining Lemma 2.3, Lemma 2.3 Corollary, Lemma 2.4 and Lemma 2.5 we obtain

Lemma 2.6. Let $i$ be a positive integer, $k$ a non-negative integer, $\Omega$ be an open set satisfying the condition $C\left(T_{0}, k+i\right)$, and let $K(x, z)$ $\in \mathscr{B}^{k+i^{-}}\left(\boldsymbol{R}^{n} \times \boldsymbol{R}^{n}\right)$.

(A) For any function $f \in C^{1}(\Omega)$ and $0<t<T_{0}$ we define $V(t, x)$ by (2.10). 
Then the following inequalities hold for any function $f$ belonging to $C^{1}(\Omega) \cap L^{p}(\Omega)$.

(i) If $0<t<T_{0}$,

$$
|V(t, x)|_{w_{p}^{k}(\Omega)} \leqq C \sum_{h=0}^{k} t^{-h}\|f\|_{L^{b}(\Omega)} .
$$

(ii) If $0<\sigma<i, 0<t<T_{0}$,

$$
|V(t, x)|_{B_{p, q}^{k+\sigma, i}(\Omega)} \leqq C \sum_{h=0}^{k}\left(t^{\sigma}+1\right) t^{-\sigma-h}\|f\|_{L^{p}(\Omega)} .
$$

(iii) If $0<\sigma<i, \sigma \leqq l, 0<T<T_{0}$,

$$
\left|\int_{0}^{T} V(t, x) t^{l-1} d t\right|_{B_{p, \infty}^{\sigma, i}(\Omega)} \leqq C\left(T^{\sigma}+1\right) T^{l-\sigma}\|f\|_{L^{p}(\Omega)}
$$

(B) Assume moreover that

$$
K(x, z)=\sum_{|\alpha|=j-1} D_{z}^{\alpha} H_{\alpha}(x, z)
$$

where $D_{z}^{\beta} H_{\alpha}(x, z) \in\left(\mathscr{B}^{k+i-}\left(\mathbb{R}^{n} \times \mathbb{R}^{n}\right)\right.$ for any $\alpha$ with $|\alpha|=j-1$ and any $\beta$ with $|\beta| \leqq j-1$, and that

$\sup H_{\alpha} \subset \mathbb{R}^{n} \times B, \quad \int H_{\alpha}(x, z) d z=0 \quad$ for any $\quad|\alpha|=j-1$.

Then, the function $V(t, x)$ given by (2.10) for $f \in C^{1}(\Omega) \cap B_{p, q}^{\tau, j}(\Omega)$ $(0<\tau<j)$ satisfies the following inequalities:

(i) If $0<(2 j-1) t<T_{0}, 0<\sigma<i$

$$
\begin{aligned}
& |V(t, x)|_{W_{p}^{k}(\Omega)} \leqq C \sum_{h=0}^{k} t^{\tau^{-h}}|f|_{B_{p, q}^{\tau, j}(\Omega)}, \\
& |V(t, x)|_{B_{p, q}^{k+\sigma, i}(\Omega)} \leqq C \sum_{h=0}^{k}\left(t^{\sigma}+1\right) t^{\tau-\sigma-h}|f|_{B_{p, q}^{\tau, j}(\Omega)}
\end{aligned}
$$

(ii) If $0<(2 j-1) T<T_{0}, l+\tau-k-\sigma \geqq 0,0<\sigma<i$, then

$$
\left|\int_{0}^{T} V(t, x) t^{t-1} d t\right|_{B_{p, q}^{k+\sigma, i}(\Omega)} \leqq C\left(T^{\sigma}+1\right) \sum_{h=0}^{k} T^{l+\tau-\sigma-h}|f|_{B_{p, q}^{\tau, j} \mathcal{G}^{(\Omega)} .}
$$

(iii) If $0<(2 j-1) T<T_{0}$ and if $l+\tau \geqq k$, then

$$
\left\{\int_{0}^{T}\left|V(t, x) t^{l}\right|_{W_{p}^{k}(\Omega)}^{q} \frac{d t}{t}\right\}^{1 / q} \leqq C \sum_{h=0}^{k} T^{l+\tau-h}|f|_{B_{p, q}^{T, j}(g)} .
$$

If $0<(2 j-1) T<T_{0}$ and if $l+\tau \geqq k+\sigma$, then 
(2. $\left.20^{\prime}\right)$

$$
\begin{aligned}
& \left\{\int_{0}^{T}\left|V(t, x) t^{l}\right|_{B_{p, q}^{q} k+\sigma, i}(\Omega) \frac{d t}{t}\right\}^{1 / q} \\
& \leqq C \sum_{h=0}^{k}\left\{T^{\sigma}+1\right\} T^{l+\tau-\sigma-h}|f|_{B_{p, q}^{\tau, j}(\Omega) .} .
\end{aligned}
$$

Proof. According to Lemma 2.7, which will be proved below, $D^{\beta} V(t, x),|\beta|=k$, is equal to the sum of functions of the form

$$
t^{-h} \int K_{h, \beta}(x, z) f(x+t z+t \Psi(x)) d z, \quad 0 \leqq h \leqq k,
$$

so that it is sufficient to prove the case where $k=0$.

First observe that

$$
\begin{aligned}
& \left|\sum_{\nu=0}^{i}(-1)^{\nu}\left(\begin{array}{l}
i \\
\nu
\end{array}\right) V(t, x+\nu y)\right| \\
& \quad \leqq C_{1}\left\{h\left(\frac{|y|}{t}\right)^{i}+h(|y|)^{i}\right\}\left\{\sum_{\nu=0}^{i} U_{j}(t, x+\nu y)\right\},
\end{aligned}
$$

where $U_{j}$ is the function given by (2.2) and (2.4), in virtue of Lemma 2.4, Lemma 2.5 and the identities

$$
\begin{aligned}
& V(t, x+\nu y) \\
& =\int K\left(x+\nu y, z-\frac{\nu y}{t}+\Psi(x)-\Psi(x+\nu y)\right) f(x+t z+t \Psi(x)) d z, \\
& =\iint \widetilde{K}\left(x+\nu y, z-\frac{\nu y}{t}+\Psi(x)-\Psi(x+\nu y), w-\frac{\nu y}{t}+\Psi(x)-\Psi(x+\nu y)\right) \\
& \times\left\{\sum_{\mu=0}^{j}(-1)^{\mu}\left(\begin{array}{l}
j \\
\mu
\end{array}\right) f\left(x+t \frac{(j-\mu) z+\mu w}{j}+t \Psi(x)\right)\right\} d z d w .
\end{aligned}
$$

This gives that

$$
\begin{aligned}
& \left\|\sum_{\nu=0}^{i}(-1)^{\nu}\left(\begin{array}{l}
i \\
\nu
\end{array}\right) V(t, x+\nu y)\right\|_{L^{p}(\Omega t, y)} \\
& \quad \leqq(i+1) C_{1}\left\{h\left(\frac{|y|}{t}\right)^{i}+h(|y|)^{i}\right\}\left\|U_{j}(t, x)\right\|_{L^{p}(\Omega) .}
\end{aligned}
$$

Combining this with (2.7), (2.8) and (2.9), we obtain (2.15), (2.18), (2.19), (2.20) and $\left(2.20^{\prime}\right) .(2.14)$ is an immediate consequence of (2.3). According to Lemma 2.4, (2.17) follows from (2.6). Finally, it follows from (2.3) and (2.22) that 


$$
\begin{aligned}
& \left|\int_{0}^{T} V(t, x) t^{l-1} d t\right|_{\substack{B_{p, \infty}^{\sigma, i}(\Omega)\\
}} \\
& \quad \leqq T^{l-\sigma} C_{2} \sup _{y}|y|^{-\sigma} \int_{0}^{T}\left\{h\left(\frac{|y|}{t}\right)^{i}+h(|y|)^{i}\right\} t^{\sigma-1} d t\|f\|_{L^{p}(\Omega)}
\end{aligned}
$$

which gives (2.16). In fact

$$
|y|^{-\sigma} \int_{0}^{T} h\left(\frac{|y|}{t}\right)^{i} t^{\sigma-1} d t \leqq \frac{i}{\sigma(i-\sigma)} .
$$

Remark 2.2. By our argument, when $K$ is independent of $x$ and $\Psi(x)$ is a constant vector, we get the inequalities (2.15), (2.16), (2.18), (2.19) and (2.20') without the factor $t^{\sigma}+1$ or $T^{\sigma}+1$.

Lemma 2.7. Let $\Omega$ be an open set with property $C\left(T_{0}, m\right)$, and $K(x, z)$ be a $C^{m}$-function. For $f \in C^{1}(\Omega)$ we define $V(t, x)$ by (2.10). Then for any multi-index $\alpha$ with $|\alpha|=m$ we have

$$
D^{\alpha} V(t, x)=\sum_{j=0}^{|\alpha|} t^{-j} V_{j, \alpha}(t, x),
$$

where

$$
\begin{aligned}
& V_{j, \alpha}(t, x)=\int K_{j, \alpha}(x, z) f(x+t z+t \Psi(x)) d z, \\
& K_{0,0}=K, \quad K_{0, \alpha}=\frac{\partial}{\partial x_{k}} K_{0, \beta}-\sum_{i=1}^{n} \frac{\partial \Psi_{i}}{\partial x_{k}} \frac{\partial K_{0, \beta}}{\partial z_{i}} \quad \text { for } \quad D^{\alpha}=D_{k} D^{\beta}, \\
& K_{j, \alpha}=(-1)^{j} \sum_{|\gamma|=j, \gamma=\alpha}\left(\begin{array}{c}
\alpha \\
\gamma
\end{array}\right) D_{z}^{\gamma} K_{0, \alpha-\gamma}(x, z) .
\end{aligned}
$$

Proof. Induction on $|\alpha|$. Let $D^{\alpha}=D_{k} D^{\beta}$, and assume that the formula valid for $D^{\beta}$. Integrating by parts, we get

$$
\begin{aligned}
D^{\alpha} & V(t, x)=D_{k}\left\{\sum_{j=0}^{|\beta|} t^{-j} V_{j, \beta}(t, x)\right\}, \\
= & \sum_{j=0}^{|\beta|} t^{-j}\left[\int \frac{\partial}{\partial x_{k}} K_{j, \beta}(x, z) f(x+t z+t \Psi)\right) d z \\
& \left.+\int \sum_{i=1}^{n} K_{j, \beta}(x, z)\left(\delta_{i k}+t \frac{\partial \Psi_{i}}{\partial x_{k}}\right) f_{i}(x+t z+t \Psi(x)) d z\right], \\
= & \sum_{j=0}^{|\alpha|-1} t^{-j} \int\left\{\frac{\partial K_{j, \beta}}{\partial x_{k}}(x, z)-\sum_{i=1}^{n} \frac{\partial \Psi_{i}}{\partial x_{k}} \frac{\partial K_{j, \beta}}{\partial z_{i}}(x, z)\right\} f(x+t z+t \Psi(x)) d z \\
& +\sum_{j=0}^{\mid \alpha_{i}^{\prime}-1} t^{-j-1} \int \frac{\partial K_{j, \beta}}{\partial z_{k}}(x, z) f(x+t z+t \Psi(x)) d z,
\end{aligned}
$$




$$
=\sum_{j=0}^{|\alpha|} t^{-j} \int K_{j, \alpha}(x, z) f(x+t z+i \Psi(x)) d z,
$$

where $\delta_{i k}$ denotes the Kronecker's delta and

$$
\mathbb{K}_{j, \alpha}=\frac{\partial}{\partial x_{k}} K_{j, \beta}-\sum_{i=1}^{n} \frac{\partial \Psi_{i}}{\partial x_{k}} \frac{\partial K_{j, \beta}}{\partial z_{i}}-\frac{\partial}{\partial z_{k}} K_{j-1, \beta}
$$

for which we obtain by the inductive assumption

$$
\begin{aligned}
K_{j, \alpha}(x, z)=\frac{\partial}{\partial x_{k}}\left\{\sum_{|\gamma|=j, \gamma=\beta}(-1)^{|\gamma|}\left(\begin{array}{l}
\beta \\
\gamma
\end{array}\right) D_{z}^{\gamma} K_{0, \beta-\gamma}\right\} \\
\quad-\sum_{i=1}^{n} \frac{\partial \Psi_{i}}{\partial x_{k}} \frac{\partial}{\partial z_{i}}\left[\sum_{|\gamma|=j, \gamma \leqq \beta}(-1)^{j}\left(\begin{array}{l}
\beta \\
\gamma
\end{array}\right) D_{z}^{\gamma} K_{0, \beta-\gamma}\right] \\
+\frac{\partial}{\partial z_{k}}\left\{\sum_{|\gamma|=j-1, \gamma \leqq \beta}(-1)^{j}\left(\begin{array}{l}
\beta \\
\gamma
\end{array}\right) D_{z}^{\gamma} K_{0, \beta, \gamma}\right\}, \\
=(-1)^{j} \sum_{|\gamma|=j, \gamma \leqq \alpha}\left(\begin{array}{l}
\alpha \\
\gamma
\end{array}\right) D_{z}^{\gamma} K_{0, \alpha-\gamma},
\end{aligned}
$$

since

$$
\begin{aligned}
K_{0, \alpha} & =\frac{\partial K_{0,3}}{\partial x_{k}}-\sum_{i=1}^{n} \frac{\partial \Psi_{i}}{\partial x_{k}} \frac{\partial K_{0, \beta}}{\partial z_{i}}, \quad\left(\begin{array}{l}
\alpha \\
\gamma
\end{array}\right)=\left(\begin{array}{l}
\beta \\
\gamma
\end{array}\right)+\left(\begin{array}{l}
\beta \\
\gamma^{\prime}
\end{array}\right), \\
\gamma^{\prime} & =\left(\gamma_{1}, \cdots, \gamma_{k}-1, \cdots, \gamma_{n}\right) .
\end{aligned}
$$

Noting that $\omega_{\alpha}(x, z)$ and $K_{j, \alpha, \beta}(x, z)$ employed in Lemma 2.1 and in Lemma 2.1 Corollary, respectively, has required properties, and combining (2.1) and (2.1') with Lemma 2.6, we can now complete the proof of Theorem 1.1. That is, we can conclude that (1.5), (1.6) and (1.7) hold for any $f \in C^{m}(\Omega) \cap W_{p}^{m}(\Omega)$, while (1.8), (1.9), and (1.10) bold for any $f \in C^{m+j}(\Omega) \cap B_{p, q}^{m+\tau, j}(\Omega)$. The additional assumption, the smoothness of $f$, car be removed in view of Theorem 1.2, which will be proved in the next section.

\section{§3. Approximation by Srmooth Functions}

To prove the approximation theorem we consider first the case where $\Omega=\mathbb{R}^{n}$ :

Lemma 3.1. Let $\rho_{1}(x)$ be a $C_{0}^{\infty}$-finction such that supp $\rho_{1} \subset B$, $\rho_{1}(x) \geqq 0$ and $\int \rho_{1}(x) d x=1$. Sei $\rho_{\nu}(x)=\nu^{n} \rho_{1}(\nu x)$. 
(i) If $f \in W_{p}^{m}\left(\mathbb{R}^{n}\right)$, then

$$
\left\|f * \rho_{\nu}\right\|_{w_{p}^{m}\left(\boldsymbol{R}^{n}\right)} \leqq\|f\|_{w_{p}^{m}\left(\boldsymbol{R}^{n}\right)}
$$

and $f * \rho_{\nu} \rightarrow f$ in $W_{p}^{n}\left(\mathbb{R}^{n}\right)$ as $\nu \rightarrow \infty$.

(ii) If $f \in B_{p, q}^{m+\tau, j}\left(\mathbb{R}^{n}\right), 0<\tau<j$, then

$$
\left\|f * \rho_{\nu}\right\|_{B_{p, q}^{m+\tau, j}\left(\boldsymbol{R}^{n}\right)} \leqq\|f\|_{B_{p, q}^{m+\tau, j}\left(\boldsymbol{R}^{n}\right)}
$$

and $f * \rho_{\nu} \rightarrow f$ in $B_{p, q}^{n+\tau, j}\left(\right.$ 㙂n$\left.^{n}\right)$ as $\nu \rightarrow \infty$.

Proof. From the relation $D^{\alpha}(f * \varphi)=f^{(\alpha)} * \varphi$ it follows that

$$
\begin{gathered}
\sum_{|\alpha| \leqq m}\left\|D^{\alpha}\left(f * \rho_{\nu}\right)\right\|_{L^{p}\left(\boldsymbol{R}^{n}\right)} \leqq \sum_{|\alpha| \leqq m} \int \rho_{\nu}(z)\left\|f^{(\alpha)}(x-z)\right\|_{L^{p}\left(\boldsymbol{R}^{n}\right)} d z \\
=\|f\|_{W_{p}^{m}\left(\boldsymbol{R}^{n}\right)} \cdot\left(f^{(\alpha)}=D^{\alpha} f\right) .
\end{gathered}
$$

Since the set of all continuous functions with compact support is dense in $L^{p}\left(\mathbb{R}^{n}\right)$, it follows that for any function $f$ belonging to $L^{p}\left(\mathbb{R}^{n}\right)$ and for any positive number $\varepsilon$ there is a positive number $\delta=\delta(f, \varepsilon)$ such that

$$
\|f(x-z)-f(x)\|_{L\left({ }^{p} \boldsymbol{R}^{n}\right)}<\varepsilon \quad \text { if } \quad|z|<\delta .
$$

Hence, taking $\nu>\max \delta\left(f^{(\alpha)}, \varepsilon\right)^{-1}$, where the maximum is taken over all multi-index $\alpha,|\alpha| \leqq m$, we obtain

$$
\left\|D^{\alpha}\left(f * \rho_{\nu}-f\right)\right\|_{L^{p}} \leqq \int \rho_{\nu}(z)\left\|f^{(\alpha)}(x-z)-f^{(\alpha)}(x)\right\|_{L^{p}(\boldsymbol{R})} d z<\varepsilon,
$$

i. e., the first part of the lemma.

(ii) With the aid of the first part and the identity $D^{\alpha}(f * \varphi)$ $=\left(D^{\alpha} f\right) * \varphi$ it is sufficient to consider the case where $m=0$. Let $f \in B_{p, q}^{\tau, j}\left(\mathbb{R}^{n}\right)$. Since

$$
\begin{aligned}
& \left\|\sum_{i=0}^{j}(-1)^{i}\left(\begin{array}{l}
j \\
i
\end{array}\right) f * \rho_{\nu}(x+i y)\right\|_{L^{p}\left(\boldsymbol{R}^{n}\right)} \\
& \quad \leqq \int \rho_{\nu}(z)\left\|\sum_{i=0}^{j}(-1)^{i}\left(\begin{array}{l}
j \\
i
\end{array}\right) f(x-z+i y)\right\|_{L^{p}\left(\boldsymbol{R}^{n}\right)} d z, \\
& \quad=\left\|\sum_{i=0}^{j}(-1)^{i}\left(\begin{array}{l}
j \\
i
\end{array}\right) f(x+i y)\right\|_{L^{t}\left(\boldsymbol{R}^{n}\right)}
\end{aligned}
$$

the inequality concerning norms follows. Consider next the function 


$$
J(y)=\left\|\sum_{i=0}^{j}(-1)^{i}\left(\begin{array}{l}
j \\
i
\end{array}\right)\left\{f * \rho_{\nu}(x+i y)-f(x+i y)\right\}\right\|_{L^{t}\left(R^{n}\right)} .
$$

From Jessen's inequality it follows that

$$
\begin{aligned}
J(y) & \leqq \int \rho_{\nu}(z)\left\|\sum_{i=0}^{j}(-1)^{i}\left(\begin{array}{l}
j \\
i
\end{array}\right)\{f(x-z+i y)-f(x+i y)\}\right\|_{L^{p}\left(\boldsymbol{R}^{n}\right)} d z \\
& \leqq 2 F_{j}(y)
\end{aligned}
$$

where

$$
F_{j}(y)=\left\|\sum_{i=0}^{j}(-1)^{i}\left(\begin{array}{l}
j \\
i
\end{array}\right) f(x+i y)\right\|_{L^{p}\left(\boldsymbol{R}^{n}\right)}
$$

also,

$$
J(y) \leqq 2^{j} \int \rho_{\nu}(z) F_{1}(-z) d z
$$

Now, for any positive number $\varepsilon$ the inequality

$$
\int_{|y| \leqq r} F_{j}(y)^{q}|y|^{-\tau q-n} d y<2^{-q-1} \varepsilon^{q}
$$

holds with some positive number $r$, since $f \in B_{p, q}^{\tau, j}\left(\mathbb{R}^{n}\right)$, and $F_{1}(-z)<\varepsilon_{1}$ for any positive number $\varepsilon_{1}$ if $|z|<\delta\left(f, \varepsilon_{1}\right)$, since $f \in L^{p}\left(\mathbb{R}^{n}\right)$, hence $J(y)$ $<2 \varepsilon_{1}$ if $\nu>\delta\left(f, \varepsilon_{1}\right)^{-1}$. Thus we have

$$
\begin{aligned}
& \left|f * \rho_{\nu}-f\right|_{B_{p, q}^{\tau, j}\left(\boldsymbol{R}^{n}\right)}^{q}=\int J(y)^{q}|y|^{-\tau q-n} d y \\
& \quad \leqq 2^{q} \int_{|y| \leqq r} F_{j}(y)^{q}|y|^{-\tau q-n} d y+2^{j q} \varepsilon_{1}^{q} \int_{|y| \geqq r}|y|^{-\tau q-n} d y \\
& \quad<\frac{\varepsilon^{q}}{2}+\frac{2^{j q} \varepsilon_{1}^{q}}{\tau q r^{\tau q}} \\
& \quad<\varepsilon^{q}
\end{aligned}
$$

taking $\varepsilon_{1}$ so small that $\varepsilon_{1}^{q}<2^{-j q-1} \tau q r^{\tau q} \varepsilon^{q}$. This completes the proof of the second part.

As an immediate consequence of Lemma 3.1 we have

Corollary 1. The approximation theorem is valid for the case $\Omega=\mathbb{R}^{n}$.

Therefore, the interpolation inequalities hold for any function $f \in W_{p}^{m}\left(\mathbb{R}^{n}\right)$ or $f \in B_{p, q}^{m+\tau, j}\left(\mathbb{R}^{n}\right)$, in particular, 


\section{Corollary 2.}

$$
\begin{aligned}
& B_{p, q}^{k+\sigma, i}\left(\mathbb{R}^{n}\right)=B_{p, q}^{m+\tau, j}\left(\mathbb{R}^{n}\right), \quad \text { if } k+\sigma=m+\tau, 0<\sigma<i, 0<\tau<j, \\
& W_{p}^{m}\left(\mathbb{P}^{n}\right) \supset B_{p, q}^{m+\tau, j}\left(\mathbb{R}^{n}\right) \supset W_{p}^{m+j}\left(\mathbb{R}^{n}\right), \quad \text { if } \quad 0<\tau<j, \\
& B_{p, 1}^{(m-i)+i, j}\left(\mathbb{R}^{n}\right) \subset W_{p}^{m}\left(\mathbb{R}^{n}\right) \subset B_{p, \infty}^{(n-i)+i, j}\left(\mathbb{R}^{n}\right), \quad \text { if } \quad 0<i<j, i \leqq m .
\end{aligned}
$$

For convenience we shall denote by $X(\Omega)$ c the set of all functions belonging to $X(\Omega)$ with compact support contained in $\Omega$, where $X(\Omega)$ is a space of functions defined in an open set $\Omega$.

Lemma 3. 2. For any open set $\Omega$

$$
\begin{aligned}
& B_{p, q}^{k+\sigma, i}(\Omega)_{c}=B_{p, q}^{m+\tau, j}(\Omega)_{c}, \quad \text { if } \quad k+\sigma=m+\tau, 0<\sigma<i, 0<\tau<j, \\
& W_{p}^{m}(\Omega)_{c} \supset B_{p, q}^{m+\tau, j}(\Omega)_{c} \supset W_{p}^{m+j}(\Omega)_{c}, \quad 0<\tau<j .
\end{aligned}
$$

Proof. Noting that $X(\Omega) \subset X\left(\mathbb{R}^{n}\right)$ if we define $f(x)$ as 0 outside of $\Omega$, and that any function $f \in X\left(\mathbb{R}^{n}\right)$ with compact support contained in $\Omega$ belongs to $X(\Omega)_{c}$, where $X=W_{p}^{m}$ or $B_{p, q}^{n+\tau, j}$, we find that the lemma follows from Lemma 3.1 Cor. 2.

For the proof of the approximation theorem for an arbitrary open set we need the following lemma:

Lemma 3. 3. Let $X$ be one of $W_{p}^{m}$ and $B_{p, q}^{m+\tau, j}$ and let $\varphi \in C_{0}^{\infty}(\Omega)$. Then $\varphi f \in X(\Omega)$, for any $f \in X(\Omega)$.

Proof. For the case $X=W_{p}^{m}$ the lemma follows from the Leibniz' formula:

$$
D^{\alpha}(\varphi f)=\Sigma\left(\begin{array}{l}
\alpha \\
\beta
\end{array}\right) D^{\beta} \varphi D^{\alpha-\beta} f .
$$

To prove the lemma for $X=B_{p, q}^{m+\tau, j}$ we first consider the case where $m=0,0<\tau<1$. Note that

$$
\begin{aligned}
& \left\|\sum_{i=0}^{j}(-1)^{i}\left(\begin{array}{l}
j \\
i
\end{array}\right)(\varphi f)(x+i y)\right\|_{L^{(0, S i, y)}} \\
& \leqq\left\|\sum_{i=0}^{j}\left(\begin{array}{l}
j \\
i
\end{array}\right)(-1)^{i} f(x+i y) \varphi(x)\right\|_{L^{b}(\Omega, y)} \\
& +\sum_{i=1}^{j}\left(\begin{array}{l}
j \\
i
\end{array}\right)\|f(x+i y)\{\varphi(x+i y)-\varphi(x)\}\|_{\left.L^{(p} \cap_{1}, y\right)}, \\
& \leqq C F_{j}(y)+C\|f\|_{L^{b}(\Omega)} h(|y|) \text {, }
\end{aligned}
$$


where $h(t)=\min (t, 1)$. This gives $\varphi f \in B_{p, q}^{\tau, j}(\Omega)$.

Consider now the case $m=0,1 \leqq \tau<j$. Assuming that the lemma for the case where $[\tau] \leqq k-1$ is already established, we shall prove the case $[\tau]=k$. Choose a furction $\psi \in C_{0}^{\infty}(\Omega)$ so that $\psi(x)$ is equal to 1 if the distance from $x$ to $\operatorname{supp} \varphi$ is not greater than $(2 j-1) \delta$, where $\delta$ is a positive number such that dist $(\operatorname{supp} \varphi, \partial \Omega) \geqq 2 j \delta, \partial \Omega=$ the boundary of $\Omega$. Then $\varphi f=\varphi \Downarrow f$ for any $f$. Let $f \in B_{p, q}^{\tau, j}(\Omega)$. When $|y| \leqq \delta$, we divide the function

$$
J(y)=\left\|\sum_{i=0}^{j}(-1)^{i}\left(\begin{array}{l}
j \\
i
\end{array}\right)(\varphi f)(x+i y)\right\|_{L^{p}\left(\Omega_{j}, y\right)}
$$

into $(j+1)$ parts:

$$
\begin{aligned}
J(y) & \leqq \sum_{i=0}^{j}\left(\begin{array}{l}
j \\
i
\end{array}\right) \|\left\{\sum_{\nu=0}^{j-i}(-1)^{\nu}\left(\begin{array}{c}
j-i \\
\nu
\end{array}\right) \varphi(x+(j-\nu) y)\right\} \\
& \times \sum_{\nu=0}^{i}(-1)^{\nu}\left(\begin{array}{l}
i \\
\nu
\end{array}\right) f(x+\nu y) \|_{L^{p}\left(\Omega_{j, y}\right)} \\
= & \sum_{i=0}^{j}\left(\begin{array}{l}
j \\
i
\end{array}\right) J_{i}(y) .
\end{aligned}
$$

$J_{j}$ is estimated by a constant times $F_{j}(y)$, hence

$$
\int_{|y| \leqq \delta}|y|^{-\tau q} J_{j}(y)^{q} \frac{d y}{|y|^{n}} \leqq C|f|_{B_{p}^{\tau,}, j_{(\xi)}}^{q}
$$

Next consider $J_{i}(y), 0<i<j$. Since $\psi(x+\nu y)=1, \nu=0, \cdots, j-1$, on the support of

$$
\sum_{\nu=0}^{j-1}(-1)^{\nu}\left(\begin{array}{c}
j-i \\
\nu
\end{array}\right) \varphi(x+(j-\nu) y)
$$

for any fixed $y$ with $|y| \leqq \delta$, we have

$$
J_{i}(y) \leqq C_{1}|y|^{j-i} G_{i}(y),
$$

where $g=\psi f$ and $G_{i}(y)=\left\|\sum_{\nu=0}^{i}(-1)^{\nu}\left(\begin{array}{l}i \\ \nu\end{array}\right) g(x+\nu y)\right\|_{L^{p}\left(\Omega_{\nu}, y\right)}$, so that

$$
\begin{aligned}
\int_{|y| \leqq \delta} J_{i}(y)^{q}|y|^{-\tau q-n} d y & \leqq C \int_{|y| \leqq \delta} G_{i}(y)^{q}|y|^{-\sigma q-n} d y, \\
& \leqq C|g|_{B_{q, q}^{q}, i(\Omega)}^{q}<\infty,
\end{aligned}
$$

in view of $g=\psi f f \in B_{p, q}^{\sigma, j}(\Omega)_{c}=B_{p, q}^{\sigma, i}(\Omega)_{c}$ for some positive $\sigma$ such that $\tau-j+i \leqq \sigma<i$. Also we get 


$$
\begin{aligned}
& \int_{|y| \leqq \delta} J_{0}(y)^{q}|y|^{-\tau q-n} d y \leqq C \int_{|y| \leqq \delta}|y|^{-\tau q+j q-n} d y\|f\|_{L^{p}(\Omega)}^{q}<\infty, \\
& \int_{|y| \geqq \delta} J(y)^{q}|y|^{-\tau q-n} d y \leqq C \int_{|y| \geqq \delta}|y|^{-\tau q-n} d y\|f\|_{L^{p}(\Omega)}^{q}<\infty,
\end{aligned}
$$

hence $\varphi f \in B_{p, q}^{\tau, j}(\Omega)_{c}$. Now, proceed to the case $m>0$. Let $f \in B_{p, q}^{m+\tau, j}(\Omega)$, and let $\alpha$ be a multi-index with $|\alpha|=m$. From Leibniz' formula it follows that

$$
\begin{aligned}
D^{\alpha}(\varphi f) & =\sum_{i=0}^{j-1} \sum_{|\beta|=i}\left(\begin{array}{c}
\alpha \\
\beta
\end{array}\right) \varphi^{(\beta)} f^{(\alpha-\beta)}+\sum_{|\beta| \geqq j}\left(\begin{array}{c}
\alpha \\
\beta
\end{array}\right) \varphi^{(\beta)} f^{(\alpha-\beta)}, \\
& =g_{0}+g_{1}+\cdots+g_{j \cdot}
\end{aligned}
$$

The first term $g_{0}$ belongs to $B_{p, q}^{\tau, j}(\Omega)_{c}$ in view of the lemma for the case just proved. Since $f^{(\beta)} \in B_{p, q}^{i+\tau, j}(\Omega) \subset B_{p, q}^{i+\sigma, j}(\Omega)$ for $|\beta|=m-i$, $\tau-i \leqq \sigma<\tau, \quad i=1, \cdots, j-1$, it follows that $g_{i} \in B_{p, q}^{i+\sigma, j}(\Omega)_{c} \subset B_{p, q}^{\tau, j}(\Omega)_{c}$ in virtue of Lemma 3.2. $f^{(\beta)} \in W_{p}^{j}(\Omega)$ for $|\beta| \leqq m-j$, hence $g_{j} \in W_{p}^{j}(\Omega)_{c}$ $\subset B_{p, q}^{\tau, j}(\Omega)_{c}$. Thus $D^{\alpha}(\varphi f) \in B_{p, q}^{\tau, j}(\Omega)_{c}$, which completes the proof of the lemma.

Now we are in pcsition to prove the approximation theorem. Our proof is due to N. G. Meyers and J. Serrin [11]. They discuss the case for $W_{p}^{m}(\Omega)$. Let $X=W_{p}^{m}$ or $B_{p, q}^{m+\tau, j}$. Let $\Omega_{\nu}$ be the open set defined by

$$
\Omega_{\nu}=\{x ; x \in \Omega,|x|<\nu, \operatorname{dist}(x, \partial \Omega)>1 / \nu\},
$$

where $\nu=1,2, \cdots, \Omega_{0}=\Omega_{-1}=$ the null set. Choose a partition of unity $\sum_{\nu=1}^{\infty} \psi_{\nu}(x)=1$ on $\Omega$ such that

$$
\text { supp } \psi_{\nu} \subset \Omega_{\nu+1}-\Omega_{\nu-1}, \nu=1,2, \cdots \text {. }
$$

Applying Lemma 3.3, we find that $\psi_{\nu} f \in X(\Omega)_{c}$ for any $f \in X(\Omega)$ and $\nu=1,2, \cdots$, so that we can choose $C_{0}^{\infty}$-function $\varphi_{\nu}$ satisfying this conditions

$$
\operatorname{supp} \varphi_{\nu} \subset\{x ;|x|<1 /(\nu+1)(\nu+2)\}, \quad \int \varphi_{\nu}(x) d x=1, \quad \varphi_{\nu}(x) \geqq 0,
$$

and

$$
\left\|\varphi_{\nu} * \psi_{\nu} f-\psi \psi_{\nu} f\right\|_{X(\Omega)} \leqq \varepsilon / 2^{\nu}, \quad \nu=1,2, \cdots,
$$

where $\varepsilon$ is an arbitrary positive number, with the help of Lemma 3.1 
and the fact that $X(\Omega)_{c} \subset X\left(\mathbb{R}^{n}\right)$ (the norm of the injection $\leqq 1$ ). Evidently, supp $\varphi_{\nu} * \psi_{\nu} f$ is contained in the set $\Omega_{v+2}-\Omega_{v-2}$, so that the series

$$
g=\sum_{\nu=1}^{\infty} \varphi_{\nu} * \psi_{\nu} f
$$

is convergent and defines a function in $C^{\infty}(\Omega)$. Furthermore, for $k=1$, $2, \cdots$, we have

$$
\begin{aligned}
\|f-g\|_{X\left(\Omega_{L}\right)} & =\left\|\sum_{\nu=1}^{k+2}\left(\varphi_{\nu} * \psi_{\nu} f-\psi_{\nu} f\right)\right\|_{X\left(\Omega_{k}\right)} \\
& \leqq \sum_{\nu=1}^{k+2}\left\|\varphi_{\nu} * \psi_{\nu} f-\psi_{\nu} f\right\|_{X(\Omega)}<\varepsilon
\end{aligned}
$$

from which we obtain, letting $k \rightarrow \infty$,

$$
\|f-g\|_{x(\Omega)} \leqq \varepsilon
$$

according to the Lebesgue monotone convergence theorem.

This completes the proof of the approximation theorem.

\section{\$4. Proof of Theorem 1.3 and Theorem 1.4}

The last two theorems can be proved as follows. First, note that for an open set $\Omega$ satisfying the condition $C\left(T_{0}, 1\right)$, for any function $f \in C^{m}(\Omega)$ and for $0<T<T_{0}$

$$
\begin{aligned}
f(x)=\int_{0}^{T} \int & M(x, z) f(x+t z+t \Psi(x)) d z \frac{d t}{t} \\
& +\int \omega_{m, 0}(x, z) f(x+T z+T \Psi(x)) d z
\end{aligned}
$$

where

$$
M(x, z)=\sum_{|\alpha|=m}(-1)^{m} D_{z}^{\alpha} \omega_{\alpha}(x, z),
$$

and $\omega_{\alpha}, \omega_{k, 0}$ are the functions given in Lemma 2.1 .

In the following part of this section we shall fix a positive integer $m$, and denote by $M$ the function given by (4.2).

Lemma 4.1. Let $\Omega$ satisfy the condition $C\left(T_{0}, m\right)$. For $0<3 T$ $<T_{0}$ and for $f \in L^{1}(\Omega)+L^{\infty}(\Omega)$ we define $E f=u$ by 
(4.3) $\quad u(t, x)= \begin{cases}\left.\int M(x, z) f(x+t z+t \Psi)\right) d z \quad \text { when } & 0<t \leqq T \\ \frac{T^{m}}{m t^{\prime \prime}} \int \omega_{m, 0}(x, z) f(x+T z+T \Psi(x)) d z, & \text { when } t>T .\end{cases}$

Then $E$ is a continuous linear operator from $L^{1}(\Omega)+L^{\infty}(\Omega)$ into $L_{\mathrm{loc}}^{1}\left(\mathbb{R}_{+} ; L^{1}(\Omega)+L^{\infty}(\Omega)\right)$, its restriction on $B_{p, q}^{\theta m}(\Omega), 0<\theta<1$ is a bounded linear operator from $B_{p, q}^{\theta m}(\Omega)$ into $W\left(q,-\theta m, L^{p}(\Omega) ; q,-(\theta-1) m\right.$, $W_{p}^{m}(\Omega)$ ), and $P E=$ the identity on $B_{p, q}^{\theta m}(\Omega)$, where

$$
(P u)(x)=\int_{0}^{\infty} u(t, x) \frac{d t}{t}
$$

Proof. The first part of the conclusion is obvious. Let $f \in C^{m}(\Omega)$ $\cap B_{p, q}^{\theta m}(\Omega)$ and let $u=E f$. For $0<t \leqq T$, integrating by parts $k$-times, we have

$$
u(t, x)=t^{k} \int_{|\beta|=k} M_{\beta}(x, z) f^{(\beta)}(x+t z+t \Psi(x)) d z
$$

where

$$
M_{\beta}(x, z)=\sum_{|\alpha|=m, \alpha=\beta}(-1)^{n-k}\left(\begin{array}{c}
m \\
k
\end{array}\right)^{-1}\left(\begin{array}{l}
\alpha \\
\beta
\end{array}\right) D_{z}^{\alpha-\beta} \omega_{\alpha}(x, z),
$$

since $M(x, z)$ is given by (4.2), while

$$
D^{\alpha}=\sum_{|\beta|=k}\left(\begin{array}{c}
|\alpha| \\
k
\end{array}\right)^{-1}\left(\begin{array}{c}
\alpha \\
\beta
\end{array}\right) D^{\beta} D^{\alpha-\beta}
$$

Combining this with Lemma 2.6, we find that

$$
\left\{\int_{0}^{T}\left\|t^{-\theta m} u(t, x)\right\|_{L^{p}(\Omega)} \frac{d t}{t}\right\}^{1 / q} \leqq C|f|_{B_{p, q}^{\theta m}(\Omega)}
$$

and that

$$
\left\{\int_{0}^{T}\left\|t^{m(1-\theta)} u(t, x)\right\|_{w_{p}^{m}(\rho)}^{q} \frac{d t}{t}\right\}^{1 / q} \leqq C_{T}|f|_{B_{p, q}^{\theta m}(\Omega)}
$$

It is easily seen that the integrals over $t \geqq T$ are estimated by a constant times $\|f\|_{L^{p}(\Omega)}$ in view of Lemma 2.6 (A). Thus we obtain for $f \in C^{m}(\Omega) \cap B_{p, q}^{\theta n}(\Omega)$

$$
\|u\|_{W\left(q,-\theta m, L^{B}(\Omega) ; q,-(\theta-1) m, W_{p}^{m}(\Omega)\right)} \leqq C\|f\|_{B_{p, q}^{\theta m}(\Omega)} .
$$


Also we have $P E f=f$ in view of (4.1). Since $C^{n}(\Omega) \cap B_{p, q}^{\theta m}(\Omega)$ is dense in $B_{p, q}^{\theta m}(\Omega)$, these isequality are valid for every $f \in B_{p, q}^{\theta m}(\Omega)$, and the lemma is proved.

Next, corsider a lemma, which is as analogue of Lemma 2.6.

Lemma 4. 2. (A) Let $\Omega$ and $K$ be as in Lemma 2.6 (A). Define the operator $A_{\varepsilon}$ by

$$
\left(A_{\varepsilon} \hat{\imath}\right)(x)=\int_{\varepsilon}^{T} t^{l-1} d t \int K(x, z) u(t, x+t z+t \Psi(x)) d z
$$

where $0<T<T_{0}, u(t, x) \in L_{*}^{q}\left(\right.$ 柦 $\left.^{+} ; L^{p}(\Omega)\right)$. (i) If $l>0$, then $A u$ $=\lim A_{\varepsilon} u$ exists in $L^{p}(\Omega)$ and

$$
\|A u\|_{L^{r}(\Omega)} \leqq C T^{l}\|u\|_{L_{*}^{q}\left(R^{+} ; L^{p}(\cdot)\right)}
$$

(ii) If $l-\sigma \geqq 0$ and if $0<\sigma<i$, then $A u=\lim A_{\varepsilon} u$ exists in $B_{p, q}^{\sigma, i}(\Omega)$ and

$$
|A u|_{B_{p, q}^{\sigma, i}\left(\varepsilon_{e}\right)} \leqq C\left(T^{l-\sigma}+T^{l}\right)\|u\|_{L_{*}^{q}\left(R^{+} ; L^{\prime}(\Omega)\right)}
$$

(B) The same facts hold where $L_{*}^{q}\left(\mathbb{R}^{+} ; L^{p}(\Omega)\right)$ is replaced by $L_{*}^{q}\left(\mathbb{R}^{+} ; B_{p, q}^{\tau, j}(\Omega)\right), 0<\tau<j$, l replaced by $l+\tau$, under the assumptions on $\Omega$ and $K$ stated in Lemma 2.6 (B).

Proof. It is surficient to prove the inequalities for any function $u(t, x)$ which is continuous $C^{j}(\Omega) \cap X(\Omega)$-valued function with compact support in $\mathbb{R}^{+}$, where $X=L^{p}$ or $B_{p, q}^{\tau, j}$, since the set of such functions is dense in $L_{*}^{q}\left(\mathbb{R}^{+} ; X(\Omega)\right)$ in virtue of the approximation theorem (see also e. g. [3] chap. 4).

Part (i) is evident in view of Lemma 2. 3.

Consider part (ii). Let $u(t, x)$ be a continuous $C^{j}(\Omega) \cap L^{p}(\Omega)$ valued function with compact support in $\mathbb{R}^{+}$. Then $f=A u$ is equal to

$$
\int_{0}^{T} t^{l-1} \int K(x, z) u(t, x+t z+t \Psi(x)) d z d t
$$

Following the proof of Lemma 2.6 (A), we have 


$$
\begin{aligned}
& \left\|\sum_{\nu=0}^{i}(-1)^{\nu}\left(\begin{array}{l}
i \\
\nu
\end{array}\right) f(x+\nu y)\right\|_{L^{p}\left(\Omega_{i}, y\right)} \\
& \quad \leq C \int_{0}^{T} t^{l-1}\left\{h\left(\frac{|y|}{t}\right)^{i}+h(|y|)^{i}\right\}\|u(t, x)\|_{L^{p}(\Omega)} d t .
\end{aligned}
$$

Thus, applying Lemma 2.2 to this integral transformation, we obtain the desired inequality (4.8), with the aid of the facts that

$$
\begin{aligned}
& \int_{0}^{T} t^{\sigma}|y|^{-\sigma} h\left(\frac{|y|}{t}\right)^{i} \frac{d t}{t} \leqq \frac{i}{\sigma(i-\sigma)} \\
& \int t^{\sigma}|y|^{-\sigma} h\left(\frac{|y|}{t}\right)^{i} \frac{d y}{|y|^{n}}=\frac{i n a}{\sigma(i-\sigma)} .
\end{aligned}
$$

The above proof also gives that the operator norm of $A_{\varepsilon}$ is not greater than $C_{1}$ which is independent of $\varepsilon$, so that the lemma follows from the following well known fact: if $\left\{A_{n}\right\}$ is a sequence of bounded linear operators from a Banach space $X$ into a Banach space $Y$ with $\left\|A_{n}\right\| \leqq C, n=1,2, \cdots$, and if $\lim A_{n} x$ exists in $Y$ for every $x$ belonging to a dense subset $S$ in $X$, then $A x=\lim A_{n} x$ exists for all $x \in X$ and $\|A\| \leqq C$.

The proof of the lemma is complete.

Finally, according to the above lemma we have

Lemma 4.3. Let $J_{\nu}$ be the operator defined by

$$
\begin{aligned}
& \left(J_{\nu} u\right)(x)=\int_{1 / \nu}^{T} \int M(x, z) u(t, x+t z+t \Psi(x)) d z \frac{d t}{t} \\
& +\frac{T^{m}}{m} \int_{T}^{\nu} t^{-m} \omega_{m, 0}(x, z) u(t, x+T z+T \Psi(x)) d z \frac{d t}{t},
\end{aligned}
$$

for $u \in L_{\mathrm{los}}^{1}\left(\boldsymbol{R}^{+} ; L^{1}(\Omega)+L^{\infty}(\Omega)\right)$. Then, considering as an operator from $L_{*}^{q,-m}\left(\boldsymbol{R}^{+} ; L^{p}(\Omega)\right)+L_{*}^{q,(1-\theta) m}\left(\boldsymbol{R}^{+} ; W_{p}^{m}(\Omega)\right)$ into $B_{p, q}^{\theta m}(\Omega)$, where $0<\theta<1$, $J_{\nu}$ converges strongly to a bounded operator $J$, and $J u=f$ if $u(t, x)$ $=f(x)$ for a.e.t. Here we assume that $\Omega$ satisfies the condition $C\left(T_{0}, m\right)$, and $T$ is a number such that $0<T<T_{0}$.

Proof. Set

$$
u=\int_{1 / \nu}^{T} \int+\int_{T}^{\nu} \int=J_{\nu}^{(1)} u+J_{\nu}^{(2)} u
$$


From Lemma 4.2 it is easy to find that $J_{\nu}^{(1)}$, regard as an operator from $L_{*}^{q,-\theta i n}\left(\mathbb{R}^{+} ; L^{p}(\Omega)\right)$ into $L^{p}(\Omega)$, converges strongly to a bounded operator. With the aid of Lemma 2.7 we can find that $J_{\nu}^{(1)}$ converges strongly when considered as an operator into $B_{p, q}^{\theta m}(\Omega)$. In the same way we see that $J_{\nu}^{(1)}$ converges strongly when considered as an operator from $L_{*}^{q, m-\theta m}\left(\mathbb{R}^{+} ; W_{p}^{m}(\Omega)\right)$ into $B_{p, q}^{\theta m}(\Omega)$, observing that, in view of (4.2),

$$
\left(J_{\nu}^{(1)} u\right)(x)=\sum_{|\beta|=m} \int_{1 / \nu}^{T} \int^{m} M_{\beta}(x, z) u^{(\beta)}(t, x+t z+t \Psi(x)) d z \frac{d t}{t},
$$

where $u^{(\beta)}(t, x)=D_{x}^{\beta} u(t, x), M_{\beta}(x, z)$ is the function given by (4.5).

Next consider the convergence of $J_{\nu}^{(2)}$. From Lemma 2.3 it follows that

$$
\begin{aligned}
\left\|J_{\nu}^{(2)} u\right\|_{L^{p}(\Omega)} \leqq C T^{m} \int_{T}^{\infty} t^{-m}\|u(t, x)\|_{L^{p}(\Omega)} \frac{d t}{t} \\
\leqq C_{1} T^{\theta m}\left\|t^{-\theta m} u(t, x)\right\|_{L_{*}^{q}\left(\boldsymbol{R}^{+} ; L^{p}(\Omega)\right)}
\end{aligned}
$$

and that $J_{\nu}^{(2)} u-J_{\mu}^{(2)} u$ tends to 0 as $\nu, \mu \rightarrow \infty$ if $u$ belongs to $L_{*}^{q,-\theta m}\left(\boldsymbol{R}^{+}\right.$; $\left.L^{p}(\Omega)\right)$. Thus $J_{\nu}^{(2)}$ converges strongly to a bounded operator as an operator from $L_{*}^{q,-\theta m}\left(\mathbb{R}^{+} ; L^{p}(\Omega)\right)$ into $L^{p}(\Omega)$. Also, from Lemma 2.6 and Lemma 2.7 it follows that $J_{\nu}^{(2)}$ converges strongly as an operator into $B_{p, q}^{\theta m}(\Omega)$. The strong convergence of $J_{\nu}^{(2)}$ as an operator from $L_{*}^{q, m-\theta m}\left(\mathbb{R}^{+} ; W_{p}^{m}(\Omega)\right)$ is proved analogously.

The last assertion is obvious in view of (4.1).

This establishes the lemma.

Now, Theorem 1. 3 follows immediately from Lemma 4.1 and Lemma 4.3, and Theorem 1.4 follows also in virtue of the following theorems due to P. Grisvard [5]:

Theorem 4.1. Let $\mathfrak{X}$ be a Banach space and let $X_{1}, X_{2}, Y_{1}$ and $Y_{2}$ be Banach spaces contained in $\mathfrak{X}$ with continuous injections.

(i) Assume that there exists a continuous linear operator $E$ from $\mathscr{X}$ into $L_{\mathrm{loc}}^{1}\left(\mathbb{R}^{+} ; \mathfrak{X}\right)$ such that its restriction on $S_{i}=S\left(p_{i}, \eta_{i}, X_{i}\right.$; $\left.p_{i}, \eta_{i}-1, Y_{i}\right)$ is a continuous linear operator from $S_{i}$ into $W\left(p_{i}, \lambda_{i} \eta_{i}\right.$, $\left.X_{i} ; p_{i}, \lambda_{i}\left(\eta_{i}-1\right), Y_{i}\right), \lambda_{i} \neq 0, i=1,2$, and that $P E f=f$ for $f \in S_{1}+S_{2}$, 
where

$$
P u=\int_{0}^{\infty} u(t) \frac{d t}{t}
$$

Then

$$
S\left(p, \theta, S_{1} ; p, \theta-1, S_{2}\right) \subset S\left(p, \eta, X_{\theta, p} ; p, \eta-1, Y_{\theta, p}\right)=S_{\theta}
$$

with continuous injection, where

$$
\begin{aligned}
& \eta=\frac{(1-\theta) \lambda_{1} \eta_{1}+\theta \lambda_{2} \eta_{2}}{(1-\theta) \lambda_{1}+\theta \lambda_{2}}, \quad \frac{1}{p}=\frac{1-\theta}{p_{1}}+\frac{\theta}{p_{2}} \\
& X_{\theta, p}=S\left(p, \theta, X_{1} ; p, \theta-1, X_{2}\right), \quad Y_{\theta, p}=S\left(p, \theta, Y_{1} ; p, \theta-1, Y_{2}\right) .
\end{aligned}
$$

(ii) Assume that there exists a continuous linear operator $J$ from $\mathcal{L}_{1}+\mathcal{L}_{2}$ into $\mathscr{X}$ such that its restriction on $\mathcal{L}_{i}$ is a continuous linear operator from $\mathcal{L}_{i}$ into $S_{i}, i=1,2$, and that $J u=f$ if $u(t)=f$ for a. e.t., where

$$
\mathcal{L}_{i}=L_{*}^{p_{i}, \lambda_{t} \eta_{i}}\left(\mathbb{R}^{+} ; X_{i}\right)+L_{*}^{p_{i}, \lambda_{i}\left(\eta_{i}-1\right)}\left(\mathbb{R}^{+} ; Y_{i}\right), \quad i=1,2
$$

Then

$$
S_{\theta} \subset S\left(p, \theta, S_{1} ; p, \theta-1, S_{2}\right)
$$

with continuous injection.

Theorem 4.2. (i) Under the same assumptions as in Theorem 4.1 (i),

$$
\left[S_{1}, S_{2}\right]_{\theta} \subset S\left(p, \eta, X_{\theta} ; p, \eta-1, Y_{\theta}\right)
$$

with continuous injection, where

$$
X_{\theta}=\left[X_{1}, X_{2}\right], \quad Y_{\theta}=\left[Y_{1}, Y_{2}\right]_{\theta} .
$$

(ii) Under the same assumptions as in Theorem 4.1 (ii),

$$
\left[S_{1}, S_{2}\right]_{\theta} \supset S\left(p, \eta, X_{\theta} ; p, \eta-1, Y_{\theta}\right)
$$

with continuous injection.

\section{References}

[1] Besov, O. V.. On a certain family of functional spaces. imbedding and continuation, Dokl. Akad. Nauk SSSR, 126 (1956), 1163-1165. (Russian)

[2] - Investigation of a family of function spaces in connection with theorems of imbedding and extension, Trudy Mat. Inst. Steklov, 60 (1961), 42-81. 
(Russian) = A. M. S. Transl. (2) 40 (1964), 85-126.

[3] Bourbaki, N., Intégration, Hermann, Paris, seconde édition, 1965.

[4] Calderón, A. P., Intermediate spaces and interpolation, the complex method, Studia Math. 24 (1964). 113-190.

[5] Grisvard, P., Commutativité de deux foncteurs d'interpolation et applications. J. Math. Pures Appl. 45 (1966), 143-206, 207-290.

[6] Il'in. V. P., The Properties of some classes of differential functions of several variables defined in an $n$-dimensional region, Trudy Mat. Inst. Steklov, 66 (1962), 227-363. (Russian)=A. M. S. Transl. (2) 81 (1969), 91-256.

[7] Komatsu, H., Fractional powers of operators II, Interpolation spaces, Pacific J. Math. 21 (1967), 89-111.

[8] Lions, J. L., Une construction d'espaces d'interpolation, C. R. Acad. Sci. Paris, 251 (1961), 1853-1855.

[9] Lions, J. L. et E. Magenes, Problemi ai limiti non omogenei, III, IV, Ann. Scuola Norm. Sup. Pisa, 15 (1961) 41-103, 311-326.

[10] Lions, J. L. et J. Peetre, Sur une classe d'espaces d'interpolation, Publ. Math. IHES, 19 (1954), 5-68.

[11] Meyers, N. and J. Serrin, $H=W$. Proc. Nat. Acad. Sci. U.S. A. 51 (6) (1964), 1055-1056.

[12] Muramatu, T., On imbedding theorems for Sobolev spaces and some of their generalization, Publ. RIMS, Kyoto Univ. 5 (1968), 393-416.

[13] - Products of fractional powers of operators, J. Fac. Sci. Univ. Tokyo, to appear.

[14] Nikol'skii, S. M., On imbedding, continuation and approximation theorems for differential functions of several variables, Uspehi Mat. Nauk, 16, No. 5 (1961), 63-114 (Russian) = Russian Math. Surveys, 16 No. 5 (1961), 55-104.

[15] Nikolsky, S. M J. L. Lions and L. I. Lizorkin, Integral representation and isomorphism properties of some classes of functions, Ann. Scuola Norm. Sup. Pisa, 19 (1965), 127-178.

[16] Nirenberg, L., On elliptic partial differential equations, Ann. Scuola Norm. Sup. Pisa, $\mathbb{1 3}$ (1959), 115-161.

[17] Taibleson, M. H., On the theory of Lipschitz spaces of distributions on Euclidean $n$-space, I: Principal properties, J. Math. Mech. 13 (1964), 407-479.

[18] Uspenskii. S. V., Properties of classes $W_{p}^{r}$ with fractional derivative on differentiable manifolds, Dokl. Akad. Nauk SSSR, 132 (1960). 60-62. (Russian)

[19] Yoshikawa, A., Remarks on the theory of interpolation spaces, J. Fac. Sci. Univ. Tokyo, 15 (1968), 209-251. 
